A B HANDLUNGEN DER DEUTSGHEN AKADEMIE DER WISSENSCHAFTEN ZU BERLIN

Klasse für Philosophie, Geschichte,

Staats-, Rechts- und Wirtschaftswissenschaften

Jahrgang 1955 Nr. 2

RAMKRISHNA MUKHERJEE

\title{
THE PROBLEM OF UGANDA
}

A Study in Acculturation 

ABHANDLUNGEN DER DEUTSCHEN AKADEMIE DER WISSENSCHAFTEN Z U B ERLIN

Klasse für Philosophic, Geschichte,

Staats-, Rechts- und Wirtschaftswissenschaften Jahrgang 1955 Nr. 2

RAMKRISHNA MUKHERJEE

\section{THE PROBLEM OF UGANDA}

A Study in Acculturation

1956

AKADEM I E-VER LAG - B E R L I N 
Vorgelegt von Hrn. Meusel in der Sitzung vom 5. Mai 1955

Zum Druck genehmigt am gleichen Tage, ausgegeben am 15. August 1956

Erschienen im Akademie-Verlag GmbH, Berlin W 8, Mohrenstraße 39

Lizenz-Nr. $202 \cdot 100 / 216 / 56$

Kartengenehmigung (K. 11) MdI. Nr. 276I

Gesamtherstellung: Druckerei „Thomas Müntzer“ Langensalza

Bestell- und Verlagsnummer: 2001/55/V/2

Preis: DM 29.-

Printed in Germany 